\title{
Prediction of protein-protein interaction types using association rule based classification
}

\author{
Sung Hee Park ${ }^{1}$, José A Reyes ${ }^{2,3}$, David R Gilbert², Ji Woong Kim¹,4 and \\ Sangsoo Kim*1
}

Address: ${ }^{1}$ Department of Bioinformatics \& Life Science, Soongsil University, Seoul, 156-743, Korea, ${ }^{2}$ School of Information Systems, Computing and Mathematics, Brunel University, Uxbridge, UB8 3PH, UK, ${ }^{3}$ Facultad de Ingeniería, Universidad de Talca, Talca, Chile and ${ }^{4}$ Equispharm Co., Ltd, Seoul, 443-766, Korea

Email: Sung Hee Park - shpark@ssu.ac.kr; José A Reyes - jareyes@dcs.gla.ac.uk; David R Gilbert - david.gilbert@brunel.ac.uk; Ji Woong Kim - phosphoros@ssu.ac.kr; Sangsoo Kim* - sskimb@ssu.ac.kr

* Corresponding author

Published: 28 January 2009

BMC Bioinformatics 2009, 10:36 doi:10.1 |86/147|-2105-10-36
Received: 19 May 2008

Accepted: 28 January 2009

This article is available from: http://www.biomedcentral.com/I47I-2/05/I0/36

(c) 2009 Park et al; licensee BioMed Central Ltd.

This is an Open Access article distributed under the terms of the Creative Commons Attribution License (http://creativecommons.org/licenses/by/2.0), which permits unrestricted use, distribution, and reproduction in any medium, provided the original work is properly cited.

\begin{abstract}
Background: Protein-protein interactions (PPI) can be classified according to their characteristics into, for example obligate or transient interactions. The identification and characterization of these PPI types may help in the functional annotation of new protein complexes and in the prediction of protein interaction partners by knowledge driven approaches.
\end{abstract}

Results: This work addresses pattern discovery of the interaction sites for four different interaction types to characterize and uses them for the prediction of PPI types employing Association Rule Based Classification (ARBC) which includes association rule generation and posterior classification. We incorporated domain information from protein complexes in SCOP proteins and identified 354 domain-interaction sites. 14 interface properties were calculated from amino acid and secondary structure composition and then used to generate a set of association rules characterizing these domain-interaction sites employing the APRIORI algorithm. Our results regarding the classification of PPI types based on a set of discovered association rules shows that the discriminative ability of association rules can significantly impact on the prediction power of classification models. We also showed that the accuracy of the classification can be improved through the use of structural domain information and also the use of secondary structure content.

Conclusion: The advantage of our approach is that we can extract biologically significant information from the interpretation of the discovered association rules in terms of understandability and interpretability of rules. A web application based on our method can be found at http://bioinfo.ssu.ac.kr/ shpark/picasso/

\section{Background}

Protein-Protein Interactions (PPIs) play a key role in many essential biological processes in cells, including signal transduction, transport, cellular motion and gene reg- ulation. The comprehensive analysis of these biological interactions has been regarded as very significant for the understanding of underlying mechanisms involved in cellular processes. 
Computational approaches for the prediction of PPI based on atomic level interactions can accurately determine the binding affinity and the specificity of binding partners. Thus, structure based prediction methods including modeling of PPI by homology modeling, threading-based methods and protein-protein docking are more accurate than methods that do not employ structure data. A major drawback of these structure-based methods is the relatively low coverage of available crystallized protein complexes in the Protein Data Bank (PDB) [1]. This is especially the case for those proteins associated with transient interactions, which is the majority of functional PPIs, and these do not form complexes stable enough for $\mathrm{x}$-ray crystallography [2]. Due to these restrictions the detailed analysis of the structure of protein complexes, specifically the area related to the interaction site between proteins, can reveal important clues for the understanding of protein functions and also characterize the specificity of these interaction regions.

The prediction of protein interaction sites has gained much attention in recent years with over 20 different methods proposed [3]. Interaction regions can be characterized by a diverse set of physico-chemical properties [46], topological properties [7] and conserved residues [8]. A variety of studies have employed different classification approaches including Support Vector Machines [9-12], Random Forests [13] and Neural Networks [14]. These studies have shown that the interfaces of interaction sites share common properties that distinguish them from the rest of the protein $[15,16,4]$. Despite their good performance in the prediction of protein interaction sites, these machine learning approaches generate final prediction models which do not provide users with explicit rules and thus result in low interpretability of the results and poor knowledge extraction capability.

The identification, analysis and characterization of different PPI types can be classified according to their life time and binding affinity into four main classes $[16,17,11]$ : obligate permanent interactions involving homo or hetero obligomers and non-obligate transient interactions involving Enzyme-inhibitor or non Enzyme-inhibitor. In obligate protein interactions, protomers which are not individually structurally stable in vivo, form permanent functional complexes that are stable and exist in their complexed form. Protomers of non-obligate interactions are independently stable and can form transient or permanent complexes. Non Enzyme-inhibitors are participants in transient interactions not involving enzymes and their protein inhibitors.

The characterization of PPI types can help for instance in the functional annotation of newly crystallized protein complexes as suggested in [17]. Several studies have been developed in this direction, focused on the discrimination of different PPI types with the aim of characterizing tran- sient and obligate protein complexes $[17,18]$. These include the statistical analysis of the interface properties [19], and the analysis from an evolutionary view of issues related to these interactions [20].

A recent computational approach [12] classified binary protein complexes into three categories (obligate interactions, non-obligate interactions and crystal packing) using six interface properties and employing Support Vector Machines (SVM). These studies [17-19,12] have improved our overall understanding of the differences between obligatory and non-obligatory or between permanent and transient interactions. Their analysis methods based on correlation drawn by the 2Ds plot of two properties has shown that a single property does not completely distinguish the different PPI types of interaction sites and the combination of more properties can give more strength to the discrimination of PPI types.

In our work we describe a computational approach for the prediction of PPI types employing association rule based classification (ARBC) $[21,22]$, which includes association rule generation and posterior classification based on the discovered rules. In a similar manner to previous approaches we investigate diverse properties associated with the interface of protein complexes. But instead of considering the entire interface area between two proteins we only consider the region associated with domain information by using the SCOP classification [23]. The use of domain profile pairs can provide better prediction of protein interactions than the use of full-length protein sequences as reported in Wojcik et al. [24]. In addition we also incorporate secondary structure information related to these domain-binding sites into our predictive approach. These features appear to be useful for the characterization and classification of binding interfaces as reported recently in Guharoy et al. [25].

The main aim of this work is to discover patterns, in the form of association rules, that characterize interaction sites in different PPI types. An important advantage of using such a classification approach is the interpretability of the final predictive model based on the analysis of the discovered set of rules. We give a detailed interpretation of discovered association rules in order to find common and specific patterns which are biologically significant and can be used to distinguish one PPI type from others. Here we focus on the prediction of four different PPI types (i.e. transient Enzyme inhibitor/Non Enzyme inhibitor and permanent homo/hetero obligomers), trying to gain more specific insights into the characterization of diverse kinds of interactions.

\section{Methods \\ Interaction Data}

We employed the same data set of non-redundant interacting protein complexes reported by [11]. The set of 147 
complexes was selected from a comprehensive set of 180 proteins taken from the PDB. 25 of these 147 complexes are involved in Enzyme-inhibitor (ENZ) interactions, 21 in non-Enzyme-inhibitor (nonENZ) interactions, 14 in hetero-obligate (HET) interaction, and 87 in homo-obligate (HOM) interactions as shown in Table 1. Proteins sharing $>20 \%$ sequence identity with a higher resolution structure of the same complex type were removed. Crystal packing structures were also eliminated by investigating the evidence in the literature that the complex occurs naturally and is stable as a dimer. NMR structures were not used, neither were mutant complexes nor structures whose resolution was > $3.0 \AA$; permanent complexes are more easily available from stable complexes by $\mathrm{x}$-ray crystallography. Transient PPIs often neither form stable complexes nor give good NMR structures. This is reflected in the small number of validated transient complexes available in the PDB.

\section{Definition of interface and dom-face}

An interface is a set of interacting atoms whose Solvent Accessible Surface Area (SASA) is decreased by $>1 \AA^{2}$ upon the formation of a complex [4]. The SASA for each atom was calculated using MSMS [26] with a probe sphere of radius $1.5 \AA$. Given a pair of interacting proteins, we define a set of interacting atoms for a single protomer as a face. An interface comprises a pair of interacting faces. We define the set of atoms comprising the face of a single domain as a dom-face. In order to calculate dom-faces, the interfaces extracted from complexes are mapped onto ranges of SCOP 1.65 domain definitions [23]. A total of 354 SCOP domains were extracted related to form the 147 protein complexes considered in our study of the different PPI types, see Table 1.

\section{Description of dom-face}

We generated 14 different physico-chemical properties and structural features to characterize each of the domfaces considered in our study including: dom-face area (dfASA), hydrophobicity $(\mathrm{HH})$, residue propensity (inPro), number of amino acids (nAA), number of atoms (nAtom), number of Secondary Structure Elements (nSSE), length of consecutive residues (LCS), number of fragments (nFrag), Size ratio of dom-face area to domain area (sRatio), Secondary Structure Elements (SSEs) content (Helix, Strand, Non-Regular) and SCOP class number (SCOPClass). Hydrophobicity and residue propensity were analyzed in the same way as Jones and Thornton [4].

The solvent accessible surface area (SASA) of a dom-face is calculated as the sum of the total decreased SASA for the interface atoms in a domain, see Equation 1. If $A$ and $B$ are two protomers in the complex $\mathrm{AB}, S A S A_{A^{\prime}} S A S A_{B}$ and $S A S$ $A_{A B}$ are SASA values for $\mathrm{A}, \mathrm{B}$, and $\mathrm{AB}$ respectively, and $n$ is the total number of interface atoms in a domain presented in protomers $\mathrm{A}$ and $\mathrm{B}$, then

$$
\text { dom-face Area }=\left(\sum_{i=1}^{n}\left(\operatorname{SASA}_{A}(i), \operatorname{SASA}_{B}(i)\right),-S A S A_{A B}(i)\right)
$$

We employed the hydrophobicity scale of Fauchere and Pliska [27] to estimate the average hydrophobicity value for each dom-face. The average hydrophobicity (HH) is calculated using Equation 2, where $H I_{A A}$ is the hydrophobicity value for each amino acid residue and $N_{A A}$ is the number of residues in a dom-face.

$$
H H=\frac{\sum_{i=1}^{l} H I A A}{N_{A A}}
$$

Residue propensity (inPro) indicates the relative frequency of different amino acid (AA) residues in dom-faces of complexes. We estimated residue propensities for all dom-faces using Equation 3 [16], where $A A P_{i}$ in Supplementary Table One [see Additional file 1] is the natural logarithm of each AA propensity and $N_{R}$ is the total number of residues in a dom-face. AA propensities for 20 amino acids over our data sets of 354 dom-faces were calculated using Supplementary Equation One [see Additional file 1].

$$
\text { inPro }=\frac{\sum_{i=1}^{n} A A P_{i}}{N_{R}}
$$

\begin{tabular}{|c|c|c|c|}
\hline Type Name & Type of Interaction & \#. of Complexes & \#. of Domains \\
\hline $\mathrm{ENZ}^{a}$ & Enzyme-inhibitors & 25 & 49 \\
\hline nonEnz ${ }^{b}$ & Non Enzyme-inhibitors & 21 & 47 \\
\hline $\mathrm{HET}^{c}$ & Hetero-obligomers & 14 & 33 \\
\hline $\mathrm{HOM}^{d}$ & Homo-obligomers & 87 & 225 \\
\hline Total & & 147 & 354 \\
\hline
\end{tabular}

Table I: Data set of protein complexes

${ }^{a} E N$ Z: Enzyme-inhibitor interactions;

bnonENZ: non-Enzyme-inhibitor interactions;

CHET: Hetero-obligate interactions;

dHOM: Homo-obligate interactions. 
In order to analyze the size of interaction sites we computed the ratio between dom-face and the whole domain area (SR) employing Equation 4.

$$
S R=\frac{A S A_{\text {dom-face }}}{A S A_{\text {domain }}}
$$

The sequence continuity in the interaction sites is described by calculating average length (number) of consecutive residues (LCS) and counting the number of consecutive residues (nFrag) in dom-faces. The SSE content is calculated by the percentages of interaction atoms located in Secondary Structure Elements (SSEs), classified using the types defined in DSSP [28]: helix, strand and non regular regions (turn, bend and loop). PPI types become the heads of association rules in ARM and the target classes in our classification. We used four different types of PPI, namely Enzyme inhibitor/Non Enzyme inhibitor as transient interaction types and homo/hetero obligomers as permanent interaction types. Other properties estimated for the diverse dom-faces analyzed were the SCOP class number at the first level of the SCOP hierarchy, the number of AA, the number of atoms and the number of SSEs present in the different interaction interfaces.

\section{Association Rule Based Classification}

The problem of predicting PPI types for a given complex of binary proteins is transformed into the task of assigning a pre-determined target class (i.e., homo/hetero obligate and non-obligate) using properties of interaction sites. We applied an efficient association rules based classification method (ARBC) to perform classification based on rules generated by Association Rule Mining (ARM). Previous studies $[21,22]$ have proposed that ARBC consistently outperforms other rule-based classifiers such as decision trees. ARBC comprises three main steps: association rule generation, pruning association rules and classification based on association rules.

\section{Association rule generation}

In our approach we employed Association Rule Mining to discover a set of frequent patterns expressed as association rules describing the relationship between properties of PPI interaction sites and PPI types. Association rules have the form $R: X \rightarrow Y[c, s]$, where $X$ and $Y$ are the body and the head of the rule respectively. $X$ and $Y$ are disjoint predicates $(X \cap Y=\varphi)$. Each $X$ and $Y$ consists of a conjunction of distinct predicates which describe properties related to interaction sites. Note that we can consider a conjunction as a set for our purposes. In our approach, the heads of all rules $Y$ are restricted to be one of the PPI types considered which are the target classes defined in this task. The strength of the association rules can be measured in terms of their support (s) and confidence (c). The support of a rule $(X \rightarrow Y)$ is the probability that the cases in a database con- tain both $X$ and $Y$. The confidence of the rule is the probability that a case contains $Y$ given that it contains $X$.

The generation of association rules was carried out employing the APRIORI algorithm [29]. We used the $10 \mathrm{~g}$ Oracle Data Miner (ODM) software which implements the APRIORI algorithm to compute the type of association rules required for our ARBC approach. We set a minimum support and confidence of $3 \%$ and $25 \%$ respectively to reduce the number of association rules generated. Association mining is not directly applicable to real valued continuous data such as some of the dom-face properties we generated. Hence we used discretisation to manipulate continuous attributes before the ARM process was executed. In this process adjacent values of continuous data were binned into a finite number of intervals.

\section{Pruning association rules}

The number of rules generated by ARM can be very large. It is necessary to prune the set of association rules by removing redundant information in order to make the classification more efficient.

Given two rules $R_{1}: X_{1} \rightarrow Y_{1}$ and $R_{2}: X_{2} \rightarrow Y_{2}$, we define:

Definition 1. The significance of a rule: $R_{1}$ is more significant than $R_{2}$ if and only if either (1) $\operatorname{conf}\left(R_{1}\right)>\operatorname{conf}\left(R_{2}\right)$ or $(2) \operatorname{conf}\left(R_{1}\right)=\operatorname{conf}\left(R_{2}\right)$ but $\sup \left(R_{1}\right)>\sup \left(R_{2}\right)$ or $(3) R_{1}$ has fewer attributes in its left hand side than $R_{2} \diamond$

Definition 2. General rule: Given two rules $R_{1}: X_{1} \rightarrow Y_{1}$ and $R_{2}: X_{2} \rightarrow Y_{2}, R_{1}$ is a general rule if and only if $X_{1} \subseteq X_{2} \diamond$

Definition 3. Overlapping rule: Given two rules $R_{1}: X_{1} \rightarrow$ $Y_{1}$ and $R_{2}: X_{2} \rightarrow Y_{2}$, then $R_{3}: X_{1} \vee X_{2} \rightarrow Y_{1}\left(\operatorname{conf}\left(R_{1}\right)\right.$, $\left.\sup \left(R_{1}\right)\right) \vee Y_{2}\left(\operatorname{conf}\left(R_{2}\right), \sup \left(R_{2}\right)\right)$ is an overlapping rule if and only if $X_{1}=X_{2}$ and $Y_{1} \neq Y_{2}$

$\diamond$ If the body of a rule $R_{1}$ is identical to the body of a rule $R_{2}$ and the head of rule $R_{1}$ is inconsistent with that of rule $R_{2}$, then an overlapping rule $R_{3}$ between two different PPI types can be identified.

Overlapping rules can be considered as common rules between two or more PPI types. On the other hand unique rules are distinctive patterns which can be used to classify interaction sites into different PPI types.

We then evaluated the following condition in order to prune the set of association rules previously generated. Given two rules $R_{1}$ and $R_{2}$, where $R_{1}$ is a general rule w.r.t. $R_{2}$, ARBC eliminate $R_{2}$ if $R_{1}$ has more significance than $R_{2}$. Sets of unique and overlapping rules were generated with the pruning procedure used in the classification. 


\section{Classification}

In the classification step we employed the pruned set of unique and overlapping rules to generate a rule profile consisting of an $m \times n$ matrix, where $m$ is the number of examples (i.e. dom-faces) and $n$ is the number of different association rules obtained after the pruning step. Each row of this matrix represents one of the dom-faces considered in our research and is associated with one of the PPI types we wish to classify. The rule profile matrix takes values of 1 or 0 depending whether the different rules are contingent or not on the respective dom-face example. A similar approach was previously employed in [30] for protein structure comparison. The rule profile matrix was generated following Algorithm 1 and then used as input to the ARBC process.

Algorithm 1 Generation of a rule profile

Input: A set of rules $\left(R_{1},<, R_{n}\right)$ and

A set of training data comprising m objects $\left(O_{1},<, O_{m}\right)$

Output: An $m \times n$ matrix, $\operatorname{RProfile}(i, j)(1 \leq i \leq m$ and $1 \leq j$ $\leq n)$

Method:

1. Sort rules in the descending order of confidence and support

2. for each rule $R_{j}$ in the descending order of the rules

for each data object $O_{i}$ in the training data

find match between $O_{i}$ and rule $R_{j}$

$$
\text { if } \operatorname{match}\left(O_{i}, R_{j}\right)
$$$$
\text { set } \operatorname{RProfile}(i, j)=1
$$

else

$$
\text { set } \operatorname{RProfile}(i, j)=0
$$

end-for

end-for
We evaluated several classification techniques for this task including Decision Trees (DT), Random Forest (RF), K Nearest Neighbor (KNN), Support Vector Machines (SVM), and Naive Bayes (NB). The WEKA machine learning library [31] was used to perform these experiments. We also performed conventional classification based only on the physicochemical properties of the different domfaces examples, without generating a set of association rules (CWAR). This was done in order to evaluate if the employment of the ARBC approach could be associated with a loss of information of some interacting complexes due, for example, to the pruning step or the discretisation of continuous value feature information. In all cases a 10 fold cross validation procedure was performed. Because the task of classification of different PPI types involves imbalanced classes (see Table 1) we utilized an over-sampling strategy, incrementing the number of instances associated with those PPI types with few examples.

\section{Results and Discussion}

\section{Analysis of dom-face Properties}

We found that $98.8 \%$ of the interaction sites studied are contained within corresponding ranges of SCOP domains. This suggests that the analysis of interaction sites based on structural domains (i.e. dom-face) does not lose interaction information.

Average values of diverse dom-face properties for different PPI types are shown in Table 2. The distribution of df-ASA for different types is presented in Supplementary Figure One [see Additional file 2]. It is possible to observe a distinct difference in the distribution of non-obligate (i.e., ENZ and nonENZ) and obligate (i.e., HET and HOM) complexes. The distribution patterns of dom-face area for ENZ are similar to those of nonENZ and the same trend occurs between HET and HOM. In the distribution of the area of interaction sites, obligate PPI types exhibit a greater variance and in general tend to have larger interaction sites than non-obligate complexes.

The average hydrophobicity $(\mathrm{HH})$ values for ENZ, nonENZ, HET and HOM are respectively 0.40, 0.37, 0.41, and 0.42 . Even though average $\mathrm{HH}$ values are similar for different PPI types, the histogram distributions of hydrophobicity (see Supplementary Figure Two [see Additional file 2]) exhibit distinctive separation patterns between non-obligate and obligate interactions. The distribution

Table 2: Average values of the properties

\begin{tabular}{cllllllll}
\hline Type & ASA $\left(\AA^{2}\right)$ & HH & inPro & nAtom & nAA & nSSE & LCS & nFrag \\
\hline ENZ & 860.42 & 0.40 & 0.596 & 121.73 & 33.71 & 11.22 & 3.3 & 12.32 \\
nonENZ & 823.06 & 0.37 & 0.530 & 106.89 & 29.59 & 12.91 & 2.5 & 12.91 \\
HET & 2237.92 & 0.41 & 0.982 & 344.26 & 82.56 & 21.35 & 3.5 & 21.35 \\
HOM & 1306.37 & 0.42 & 0.262 & 184.55 & 48.14 & 13.00 & 2.9 & 16.78
\end{tabular}


of HH for ENZ is similar to nonENZ and that of HET is similar to HOM.

We note that Arg, His, Tyr, Gln and Trp exhibit higher propensities than other amino acids, while Gly has a low propensity in our analysis. Average residue propensities are shown in Table 2. HET has the highest residue propensity and HOM the lowest. We also analyzed the top four frequent residues for each interaction type calculating the sum of ASA for each amino acid (results in Supplementary Figure Three [see Additional file 2]). Hydrophobic residues including Leu, Ala, and Val frequently occur in types HET and HOM. The charged residue Glu also appears frequently in HET. In nonENZ, charged residues including Asp, Glu, Lys, and Arg are present in the top four frequent residues.

ENZ includes not only some polar residues Ser and Tyr but also the charged residue Glu. We observed that the charged residues occur very frequently in all interaction types and appear dominantly in HET. Trp, Cys, and Met rarely occurred in interface area through all types.

The average values of the size ratio between dom-face area and domain area for ENZ, nonENZ, HET, and HOM are $27.03,20.67,31.94$, and 23.26 respectively as shown in Table 2. The distribution of size ratio is shown in Supplementary Figure Four [see Additional file 2]. We observed that $92 \%$ of dom-faces are smaller than a half of their domain sizes based on the calculation of ASA values. The average length of consecutive residues (LCS) are 3.3, 2.5, 3.5 and 2.9 for ENZ, nonENZ, HET, and HOM respectively as shown in Table 2.

The average distribution of SSE elements (helix, strand and non-regular regions) for different PPI types is shown in Figure 1. We have seen that interaction sites are mostly composed of non-regular regions followed by helix and strand regions. ENZ contains $64.15 \%$ of non-regular regions, which is the highest percentage. Helix content are greater than $36 \%$ in types nonENZ, HET and HOM but are less than $17 \%$ in ENZ. Strand content for all types are less than $20 \%$ and HET exhibits the lowest value (13.72\%).

The variation in the number of amino acids (nAA) is similar to that for the number of atoms (nAtom). Average values for nAtom, nAA, nSSE and nFrag are shown in Table 2. We found that values for Types HET and HOM are higher than for Types ENZ and nonENZ in all these categories. The distribution of these properties (results shown in Supplementary Figure Seven [see Additional file 2]) for ENZ is similar to nonENZ.

\section{Classification of PPI types}

We were able to discover a total of 1,168 rules describing associations by employing ARM. After the pruning stage a
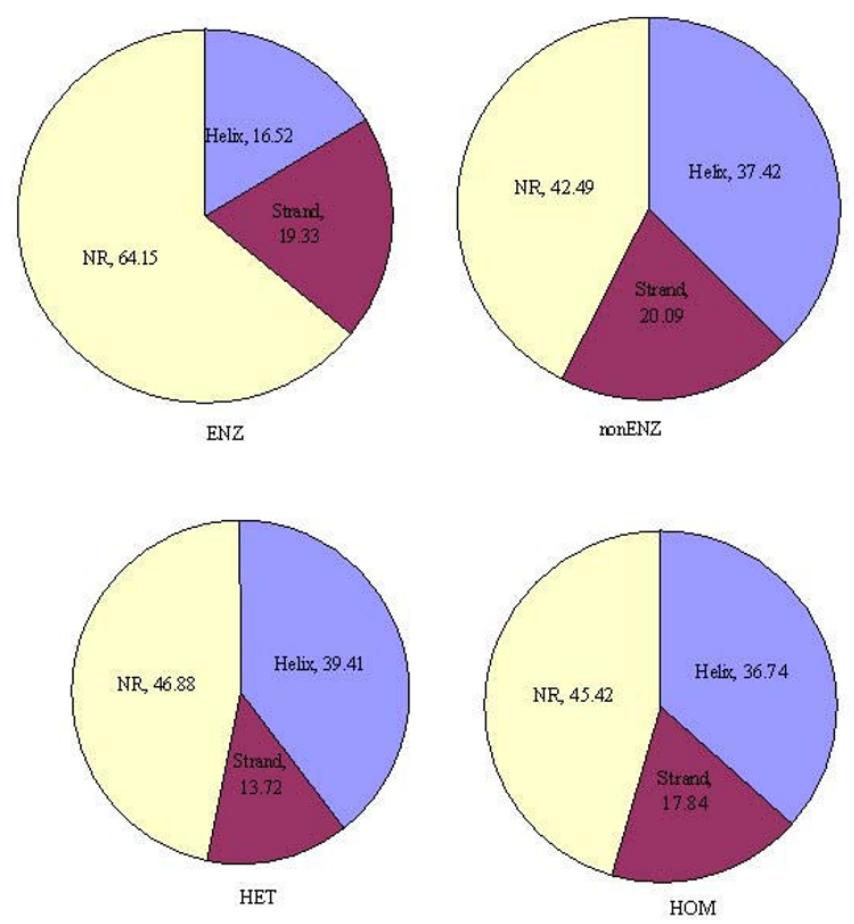

Figure I

Distribution of SSE content. The average distribution of SSE content is distinctive among different PPI types. More than $40 \%$ of atoms in interaction sites for all PPI types are positioned in non-regular regions. Interaction sites contain higher portion of non-regular regions than those of helix and strand regions. Especially, less than $20 \%$ of interaction sites are composed of strands.

total of 157 association rules [see Additional file 3] were selected for the classification process. The number of rules associated with types ENZ, nonENZ, HET and HOM are $65,49,19$, and 24 respectively (Table 3 ). A total of 58 of these are unique, i.e. rules exclusively associated with just one PPI type. The remaining 99 rules are overlapping (non-unique) rules related to two or more PPI types. We are interested in this distinction because unique rules appear to be related to specific characteristics of PPI types, whilst overlapping rules can be related to common attributes of different interaction types or for instance to distinctive properties between obligate and non-obligate interactions.

The performance for different classification methods measured as total accuracy over 10 fold cross validation for ARBC is shown in Table 4. Additionally we performed classification based on the physicochemical properties of the different dom-faces(CWAR), and also ARBC classification based on a rule profile generated using only the set of 58 unique rules discovered (UR). Performance results for these approaches are also given in Table 4 . We have seen that in all these cases SVM exhibited the best performance 
Table 3: The number of association rules discovered for each PPI type

\begin{tabular}{|c|c|c|c|c|}
\hline Type & \#. of Domains ${ }^{a}$ & \#. of Rules ${ }^{b}$ & Unique Rulesc & Overlapping Rules \\
\hline ENZ & 49 & 65 & $34(52.31 \%)$ & 31 (47.69\%) \\
\hline nonENZ & 47 & 49 & $16(32.65 \%)$ & $33(67.35 \%)$ \\
\hline HET & 33 & 19 & 7 (36.84\%) & $12(63.16 \%)$ \\
\hline HOM & 225 & 24 & I (4.17\%) & $23(95.83 \%)$ \\
\hline Total & 354 & 157 & 58 (36.94\%) & 99 (63.06\%) \\
\hline
\end{tabular}

\#. of Domains: A number of domains in each PPI type;

b\#. of Rules: A number of association rules discovered for each PPI type;

Unique Rules: A number of association rules associated with just one PPI type;

dOverlapping Rules: A number of rules of which bodies are identical to those of rules in other types.

among diverse classifiers studied, reaching over $99 \%$ accuracy in some cases. However this high accuracy suggests that overfitting problems are associated with the use of SVM. The other classification approaches evaluated still exhibit a high accuracy with the exception of NB. The performance reached by them is comparable to that previously reported in [12] although not exactly the same instances and features were employed. Additionally we observed that there was no significant appreciable difference between the performance of ARBC and CWAR in most of the situations, although it seems that CWAR performed slightly better than ARBC.

These results strongly suggest that ARBC performs competitively with conventional classification approaches for this task, and consequently the use of ARBC does not involve an important loss of information derived from ARM. The performance of ARBC using only unique rules clearly decreased for all classification methods evaluated, although maintaining an acceptable accuracy of near or over $90 \%$ in most of the cases. This suggest that unique

Table 4: Accuracy for difference classification methods

\begin{tabular}{|c|c|c|c|c|c|}
\hline Methoda & DT & RF & $\mathrm{KNN}$ & SVM & NB \\
\hline \multicolumn{6}{|l|}{ All data!: } \\
\hline$A R B C^{b}$ & 0.924 & 0.968 & 0.943 & 0.999 & 0.476 \\
\hline$C W A R^{c}$ & 0.926 & 0.971 & 0.978 & 0.999 & 0.531 \\
\hline$U R^{d}$ & 0.873 & 0.933 & 0.893 & 0.970 & 0.519 \\
\hline \multicolumn{6}{|l|}{ No SSE data ${ }^{2}:$} \\
\hline ARBC_WO_SSEe & 0.917 & 0.951 & 0.936 & 0.992 & 0.451 \\
\hline CW AR_WO_SSEf & 0.927 & 0.970 & 0.979 & 0.988 & 0.492 \\
\hline UR_WO_SSEg & 0.800 & 0.850 & 0.800 & 0.890 & 0.483 \\
\hline
\end{tabular}

aMethod represents different classification methods such as Decision Tree (DT), Random Forest (RF), K Nearest Neighbor(KNN), Support Vector Machine (SVM) and Naive Bayes (NB);

bARBC: Association rule based classification;

CCW AR: Classification based on physicochemical properties; dUR: ARBC classification using 58 unique association rules;

e, $f, g$ : Data sets with exclusion of SSE content from All data';

'All data: Data sets including SSE content;

${ }^{2}$ No SSE data: Data sets without inclusion of SSE content. rules can be influential in classifying most of the PPI types considered in our study and that overlapping rules are important to improve the accuracy of the classification task. It is important to emphasize that the aim of our research is focused on the advantage of interpretability of the discovered rules rather than the optimization of the classification task.

We further investigated the influence of SSE information on the classification of PPI types. We evaluated three different data sets without using the secondary structure elements of proteins, including ARBC_WO_SSE, CWAR_WO_SSE and UR_WO_SSE. Each of the two rule profiles in this case contains a total of only 135 association rules and 43 unique rules. Results for these evaluations are also highlighted in Table 4. It was found that in all cases the performance of diverse classifiers tended to decrease when SSE data was omitted, although only a slightly reduction is observed in most of the classifiers evaluated. Interestingly the major decrement in performance was observed when employing UR_WO_SSE, with accuracies of less than $90 \%$ for all classifiers including SVM. These results strongly suggest that SSE content in interaction sites could have an important role in the discrimination of different PPI types for both approaches including ARBC and CWAR.

This implies that the average confidences of the rule sets that include this SSE content information may be higher than those without it. There were $14.01 \%$ (22 out of 157) such rules that included SSE content information and their average confidence was 0.533 (Table 5). When we considered the top 31 rules that are covered by $20 \%$ of all the rules, their confidence was 0.642 . Among them, $42 \%$ (13 out of 31) contained SSE information with an average confidence of 0.661. The SSE content rules were enriched among those rules exhibiting higher confidences. The same trend was also seen with unique rules: while the average confidence of 58 unique rules was 0.536 , that of the 16 unique SSE rules was 0.622 . Here we infer that SSE content in interaction sites is a significant feature that permits reliable classification of the interaction types. 


\section{Interpretation of Discovered Association Rules} Determination of Important Rules

To select a set of informative and discriminative rules for the extraction of knowledge, most of the existing approaches rank the association rules based on the confidence value of a individual rule. A strong rule which is highly confident and represents general knowledge, may not be a good discriminative rule for the classification. Instead, a better measure of the importance of a rule should include the following factors considered together: correlation between a property and a class, the degree of classification power, confidence and support, top K coverage and uniqueness of a rule. As noted in the previous section, the inclusion of the SSE content information in our ARBC approach has a positive effect on the classification accuracy (Table 4). The importance of a rule can be quantified by integrating the various factors including the SSE content information. We defined a importance factor (I in Tables 6 and 7) by an average value of all the factors. In order to illustrate the informativeness of the rules in understanding interface features, some representative rules within the top 30\% (ranked higher than 48) of I are listed in Table 6 . The list was complemented by some other rules ranked below 48 in order to explain overlapping rules and compare association rules to rules generated from a decision tree. Similarly, rules describing the ENZ type with varying different structural features are listed in Table 7. Rules in Tables 6 and 7 are sorted by Type and I.

We have shown that the interaction sites were dominated by non-regular region: especially for ENZ interactions, almost $\frac{2}{3}$ of the sites in average were composed of nonhelix and non-beta strand regions (Figure 1). This is manifested in rules 29 (Table 7), 1, 4 and 6, all of which require $50-80 \%$ content of non-regular regions to be classified as ENZ. Some of the rules containing negation predicates are strong indicators of certain interaction types. For example, "Nohelix " and "Nostrand " in the interaction sites imply ENZ (Rule 29) and nonENZ (Rules 7, 12 and 15), respectively. HET is characterized by relatively

Table 5: Analysis of SSE content rules over different subsets

\begin{tabular}{cccccc}
\hline Subset & \#. of rules & Fraction(\%) & $\operatorname{conf}_{1}^{a}$ & \#. of SSE rules & conf ${ }_{2}^{b}$ \\
\hline SSE $^{c}$ & 22 & $14.01 \%$ & 0.533 & - & - \\
TOPK $^{d}$ & 31 & $19.75 \%$ & 0.642 & 13 & 0.661 \\
Unique $^{e}$ & 58 & $36.94 \%$ & 0.536 & 16 & 0.622 \\
\hline
\end{tabular}

${ }^{a}$ con $f_{1}$ : Average confidence of a rule subset;

${ }^{b}$ con $f_{2}$ : Average confidence of SSE content rules in a rule subset;

CSSE: Association rules encoding SSE content;

dTOPK: Top K rules covering top $20 \%$ in confidence;

eUnique: Unique rules. small portions of strands (Rules 18, and 19) and "Nostrand " (Rule 24). It is also observed that rules containing such SSE content information conjuncted with other properties (Rules 29, 7, 12, 15 and 24 in Figure 2) or combined with other rules (Figure 3(a), (b) and 3(c)) become stronger discriminators for classifying PPI types than rules containing only SSE content information (Rules 1, 2, 4, 6, $14,18,19$ and 21 in Figure 2). We note that some rules (Rules 29 and 7 in Figure 2) containing SSE information with SCOP classes are the most discriminative and informative in order to characterize ENZ and nonENZ.

\section{Inference of Subtypes}

Some rules which share the same sets of properties but differ in their value ranges or have other properties can be effective in order to compare features of different interaction types or to identify subtypes in a PPI type. For example, among the top 30\% rules, Rules 38 (Table 7) and 16 (Table 6) describe types ENZ and nonENZ respectively, using the same set of properties such as number of atoms and df-ASA. However, their values imply that the interaction sites of nonENZ (Rule 16) are larger than those of ENZ (Rule 38). The ranges of size scales of interaction sites in ENZ are presented in Rules 35, 38 and 46 (Table 7) that share the same set of properties but differ in their values. The overall size of interaction sites in ENZ are described by Rule 38 with the highest confidence among those rules encoding the size of interaction sites. These are interesting cases where the structural difference between types can be directly inferred and subtypes of a PPI type can be derived by grouping different features of interaction sites. We deduced five subtypes of ENZ and a hierarchical tree (Figure 4) to account for those subtypes. We compiled a list of representative association rules (Table 7 ) to show structural features different among these subtypes.

We note that interaction sites of enzymes are distinguished from those of inhibitors in enzyme-inhibitor complexes. Interaction sites for inhibitors are relatively small, i.e., mainly < $1000 \AA^{\wedge} 2$ (Rules 34, 35, 37, 38 and 46 ), and are made up of strands (Rule 41) and mostly non-regular regions (Rules 1,4 and 6) without helix content (Rule 3, 28, 29, 30, 32, and 33) which is very informative in order to characterize inhibitors. Remarkably Rules 30 and 28 generalize common features of inhibitors with respect to the size of interaction sites and SSE content. As Rule 29 was considered to be very discriminative to differentiate ENZ from other types, it can depict characteristics of a small group of inhibitors with indicating that inhibitors in SCOP class 7 do not contain helix in interaction sites (Figure 3(a), (b) and 3(c)).

In contrast, enzymes have larger interaction sites than their inhibitors and form mixtures of helices and strands 
Table 6: Representative examples of association rules for each type

\begin{tabular}{|c|c|c|c|c|c|c|c|c|c|c|c|}
\hline$\#^{a}$ & $\mathrm{O}^{b}$ & Rule description ${ }^{c}$ & Type & Confe & Supp $f$ & $\mathrm{Cg}$ & $\mathrm{G}^{h}$ & $\mathrm{~K}^{i}$ & Uj & $\mathrm{S}^{k}$ & II \\
\hline I & 3 & If 77.3 I $\leq$ Loop $<80.56$ & ENZ & 0.811 & 0.032 & I & 0.214 & I & 1 & I & 0.722 \\
\hline 2 & 8 & If $\mid 7.57 \leq$ Helix $<20.87$ & ENZ & 0.545 & 0.032 & I & 0.102 & $\mathrm{I}$ & I & 1 & 0.668 \\
\hline 3 & 9 & If SCOPClass = 7 & ENZ & 0.725 & 0.053 & I & 0.184 & I & I & - & 0.660 \\
\hline 4 & 26 & If $67.59 \leq$ Loop $<70.83$ & ENZ & 0.526 & 0.032 & - & 0.048 & I & 1 & 1 & 0.601 \\
\hline 5 & 28 & If $46 \mathrm{I} .83 \leq \mathrm{df}-\mathrm{ASA}<68 \mathrm{I} .42$ AND $2.3 \leq \mathrm{LCS}<2.73$ & ENZ & 0.625 & 0.032 & - & 0.120 & I & 1 & - & 0.555 \\
\hline 6 & 37 & If $57.87 \leq$ Loop $<6$ I.II & ENZ & 0.467 & 0.037 & - & 0.045 & - & 1 & 1 & 0.510 \\
\hline 7 & 2 & If SCOPClass $=$ I AND $12.25 \leq \mathrm{nFrag}<16$ AND NoStrand & nonENZ & 0.882 & 0.032 & I & 0.250 & I & 1 & I & 0.738 \\
\hline 8 & II & If $.66 \leq$ inPro $<.87$ & nonENZ & 0.597 & 0.042 & I & 0.129 & I & 1 & - & 0.628 \\
\hline 9 & 15 & If $26.74 \leq \mathrm{nAA}<35.32$ AND $901.0 \mathrm{I} \leq \mathrm{df}-\mathrm{ASA}<\mathrm{I} 120.6$ & nonENZ & 0.556 & 0.032 & I & 0.133 & I & 1 & - & 0.620 \\
\hline 10 & 18 & If SCOPClass = I AND I.87 <= LCS < 2.39 & nonENZ & 0.545 & 0.032 & I & 0.137 & I & 1 & - & 0.619 \\
\hline II & 20 & If $1.43 \leq \mathrm{LCS}<\mathrm{I} .87$ & nonENZ & 0.556 & 0.042 & I & 0.074 & I & 1 & - & 0.612 \\
\hline 12 & 21 & If NoStrand AND I.87 $\leq$ LCS $<2.3$ & nonENZ & 0.515 & 0.037 & - & 0.113 & I & 1 & 1 & 0.611 \\
\hline 13 & 36 & If 58. II $\leq$ ASAPR $<59.52$ & nonENZ & 0.476 & 0.032 & 1 & 0.065 & - & 1 & - & 0.515 \\
\hline 14 & 38 & If 4 I.67 $\leq$ Loop $<44.91$ & nonENZ & 0.423 & 0.032 & - & 0.046 & - & 1 & 1 & 0.500 \\
\hline 15 & 40 & If SCOPClass = I AND NoStrand & nonENZ & 0.484 & 0.064 & - & 0.074 & - & & 1 & 0.406 \\
\hline 16 & 46 & If $125.14 \leq$ nAtom $<165.52$ AND $901.01 \leq$ df-ASA $<$ I 120.6 & nonENZ & 0.412 & 0.037 & - & 0.050 & - & 1 & - & 0.375 \\
\hline 17 & 64 & If $.42 \leq \mathrm{HH}<.44$ & nonENZ & 0.347 & 0.037 & - & 0.009 & - & 1 & - & 0.348 \\
\hline 18 & 5 & If $7.78 \leq$ Strand $<10.27$ & HET & 0.660 & 0.037 & I & $0.14 \mid$ & I & 1 & 1 & 0.691 \\
\hline 19 & 7 & If $2.8 \leq$ Strand $<5.29$ & HET & 0.565 & 0.037 & 1 & 0.089 & I & 1 & 1 & 0.670 \\
\hline 20 & 12 & If $205.9 \leq$ nAtom $<246.28$ & HET & 0.574 & 0.037 & $\mathrm{I}$ & 0.143 & $\mathrm{I}$ & 1 & - & 0.626 \\
\hline 21 & 25 & If 44.9 I $\leq$ Loop $<48.15$ & HET & 0.479 & 0.037 & $\mathrm{I}$ & 0.110 & - & 1 & I & 0.604 \\
\hline 22 & 32 & If $3.6 \leq \mathrm{LCS}<4.03$ & HET & $0.46 I$ & 0.037 & 1 & 0.100 & - & 1 & - & 0.520 \\
\hline 23 & 33 & If $.44 \leq \mathrm{HH}<.46$ & HET & 0.467 & 0.045 & I & 0.070 & - & 1 & - & 0.516 \\
\hline 24 & 63 & If SCOPClass = I AND NoStrand & HET & 0.282 & 0.037 & - & 0.074 & - & - & I & 0.348 \\
\hline 25 & 31 & If SCOPClass $=3$ AND $2.3 \leq$ LCS $<2.73$ & HOM & 0.470 & 0.033 & I & 0.100 & - & 1 & - & 0.521 \\
\hline 26 & 98 & If $3.17 \leq \mathrm{LCS}<3.6$ & HOM & 0.337 & 0.035 & - & 0.034 & - & - & - & 0.135 \\
\hline 27 & 133 & If $26.74 \leq \mathrm{nAA}<35.32$ & HOM & 0.237 & 0.039 & - & 0.041 & - & - & - & 0.106 \\
\hline
\end{tabular}

Representative examples of 27 rules within top $30 \%$ are listed by sorting Columns Type and I. Rules of which order is below 48 are added for explaining overlapping rules and the comparison to rules produced from a decision tree.

\#: Rule identifier;

bO: Order of a rule ranking by importance factor;

'Rule description: The body of a rule;

¿Type: The head of a rule representing a PPI type;

eConf: Confidence of a rule;

f Supp: Support of a rule;

gC: Rules selected from correlation-based feature subset selection [32];

${ }^{h} G$ : The worth of a rule by measuring the gain ratio [33] with respect to PPI types;

iK: Top $\mathrm{K}$ rules ranked within top $30 \%$;

jU: Unique rules;

kS: SSE content rules;

I: Importance factor of a rule calculated by an average of all factors such as Conf, Supp, C, G, K, U and S; "-" is replaced with value 0 when the importance factor was calculated.

in interaction sites (Rules 40, 48, 49, 50 and 51). Both Rules 33 and 40 show that enzymes (Rule 40) have SSEs twice as many as inhibitors (Rule 33). This indicates that both enzymes and inhibitors may contain mainly strands as regular SSEs in interaction sites since enzymes are included in SCOP class 2 (mainly $\beta$ ) and inhibitors do not contain helices in interaction sites. This suggests that non regular regions and beta strands are mainly involved in the interfaces of enzyme-inhibitor interactions. Such extracted information can be useful for the prediction of interaction sites for enzyme-inhibitor complexes. This observation is demonstrated by some small inhibitors in Type ENZ_A (1tabi_,2ptci_, and 4sgbi_) and Type ENZ_B (1mcti_). Those inhibitors interact with enzymes in Type ENZ_B. The enzymes described by Rules 40,41 and 43 are included in SCOP superfamily trypsin-like serine proteases (2.47.1) and the inhibitors are mainly in SCOP class 7 which is composed of small proteins dominated by metal ligand, heme, and disulfide bridges.

It is possible in a similar way to infer subtypes of other PPI types. Among PPI types, ENZ has plenty of rules (a total of 
Table 7: Representative examples of ENZ type presenting different structural features

\begin{tabular}{|c|c|c|c|c|c|c|c|c|c|c|c|}
\hline \# & O & Rule description & Subtype & Conf & Supp & $\mathrm{C}$ & G & $\mathrm{K}$ & 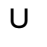 & $S$ & 1 \\
\hline 28 & 24 & If NoHelix & ENZ_A, ENZ_B, ENZ_C & 0.508 & 0.069 & - & 0.058 & 1 & 1 & I & 0.606 \\
\hline 29 & 1 & If SCOPClass = 7 AND NoHelix & ENZ_A, ENZ_B & 1.000 & 0.032 & 1 & 0.315 & 1 & 1 & I & 0.764 \\
\hline 30 & 17 & If $461.83 \leq \mathrm{df}-\mathrm{ASA}<681.42$ AND NoHelix & ENZ_A, ENZ_B & 0.593 & 0.037 & - & 0.085 & 1 & 1 & 1 & 0.619 \\
\hline 31 & 39 & If $461.83 \leq \mathrm{df}-\mathrm{ASA}<681.42$ & ENZ_A, ENZ_B & 0.477 & 0.111 & 1 & 0.076 & - & - & - & 0.416 \\
\hline 32 & 16 & If NoHelix AND nFrag $<4.75$ & ENZ_A & 0.612 & 0.032 & - & 0.076 & 1 & 1 & I & 0.620 \\
\hline 33 & 19 & If $4.75 \leq$ nSSE $<6.62$ AND NoHelix & ENZ_A & 0.588 & 0.032 & - & 0.072 & 1 & 1 & I & 0.538 \\
\hline 34 & 51 & If $461.83 \leq \mathrm{df}-\mathrm{ASA}<681.42$ AND $4.75 \leq \mathrm{nSSE}<6.62$ & ENZ_A & 0.417 & 0.032 & - & 0.018 & - & 1 & - & 0.367 \\
\hline 35 & 77 & If $44.38 \leq$ nAtom $<84.76$ AND $461.83 \leq \mathrm{df}-\mathrm{ASA}<681.42$ & ENZ_A & 0.396 & 0.058 & - & 0.023 & - & - & - & 0.159 \\
\hline 36 & 34 & $\begin{array}{l}\text { If } 9.58 \leq \mathrm{nAA}<18.16 \text { AND } 44.38 \leq \mathrm{nAtom}<84.76 \text { AND } 461.83 \leq \mathrm{df}-\mathrm{ASA}< \\
681.42\end{array}$ & ENZ_A & 0.500 & 0.032 & - & 0.045 & 1 & 1 & - & 0.515 \\
\hline 37 & 60 & If $18.16 \leq \mathrm{nAA}<26.74$ AND $44.38 \leq \mathrm{nAtom}<84.76$ & ENZ_A & 0.357 & 0.032 & - & 0.015 & - & I & - & 0.351 \\
\hline 38 & 10 & If $84.76 \leq$ nAtom $<125.14$ AND $461.83 \leq$ df-ASA $<681.42$ & ENZ_B & 0.617 & 0.053 & 1 & 0.145 & 1 & 1 & - & 0.636 \\
\hline 39 & 13 & If $12.66 \leq$ sRatio $<15.06$ AND $46 \mid .83 \leq$ df-ASA < 681.42 & ENZ_B & 0.600 & 0.032 & 1 & 0.113 & 1 & I & - & 0.624 \\
\hline 40 & 14 & If $461.83 \leq \mathrm{df}-\mathrm{ASA}<681.42$ AND $10.38 \leq \mathrm{nSSE}<12.25$ AND SCOPClass $=2$ & ENZ_B & 0.857 & 0.032 & - & 0.230 & 1 & I & - & 0.624 \\
\hline 41 & 27 & $\begin{array}{l}\text { If SCOPClass = } 2 \text { AND } 461.83 \leq \mathrm{df}-\mathrm{ASA}<681.42 \text { AND } 84.76 \leq \text { nAtom }< \\
125.14\end{array}$ & ENZ_B & 0.789 & 0.032 & - & 0.176 & 1 & I & - & 0.599 \\
\hline 42 & 35 & If $10.38 \leq$ nSSE $<12.25$ AND $12.25 \leq$ nFrag $<16$ & ENZ_B & 0.500 & 0.032 & - & 0.043 & 1 & I & - & 0.515 \\
\hline 43 & 73 & If $84.76 \leq$ nAtom $<125.14$ AND SCOPClass $=2$ & ENZ_B & 0.408 & 0.042 & - & 0.043 & - & - & - & 0.164 \\
\hline 44 & 114 & If $84.76 \leq$ nAtom $<125.14$ AND $26.74 \leq$ nAA $<35.32$ & ENZ_B & 0.307 & 0.037 & - & 0.024 & - & - & - & 0.123 \\
\hline 45 & 109 & If $681.42 \leq \mathrm{df}-\mathrm{ASA}<90 \mathrm{I} .0 \mathrm{I}$ & ENZ_C & 0.317 & 0.048 & - & 0.013 & - & - & - & 0.126 \\
\hline 46 & 137 & If $84.76 \leq$ nAtom $<125.14$ AND $681.42 \leq \mathrm{df}-\mathrm{ASA}<901.0 \mathrm{I}$ & ENZ_C & 0.252 & 0.032 & - & 0.009 & - & - & - & 0.098 \\
\hline 47 & 146 & If SCOPClass $=4$ & ENZ_C & 0.221 & 0.042 & - & 0.011 & - & - & - & 0.091 \\
\hline 48 & 101 & If 35.32901 .01 nAA $<43.9$ AND I $25.14 \leq$ nAtom $<165.52$ & ENZ_D & 0.323 & 0.032 & - & 0.041 & - & - & - & 0.132 \\
\hline 49 & 130 & If SCOPClass $=3$ & ENZ_D & 0.238 & 0.069 & - & 0.016 & - & - & - & 0.108 \\
\hline 50 & $|4|$ & If $901.01 \leq \mathrm{df}-\mathrm{ASA}<1 \mathrm{I} 20.6$ & ENZ_D & 0.207 & 0.032 & - & 0.050 & - & - & - & 0.096 \\
\hline 51 & 54 & If $\mathrm{I} \mid 20.6 \leq \mathrm{df}-\mathrm{ASA}<1340.19$ & ENZ_E & 0.392 & 0.042 & - & 0.018 & - & 1 & - & 0.363 \\
\hline
\end{tabular}

Abbreviation of column names is the same as that of Table 6.

The ENZ subtypes are defined in Figure 4. Note that ENZ_B includes both inhibitors and enzymes while the others are exclusively formed by inhibitors (e.g. ENZ_A, ENZ_C and ENZ_E) or enzymes (e.g. ENZ_D). 


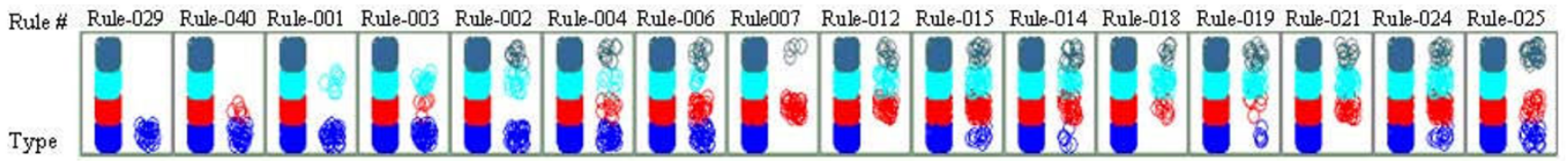

ENZ nonENZ HET HOM

Figure 2

A scatter Plot matrix for PPI types and association rules. This scatter plot matrix shows clusters as collection of points separated by association rules encoding SSE content information or a SCOP class. Different colors of the left in each plot (a cell) correspond to four PPI types. The right of a plot area presents the distribution of points met with a rule on the head of a cell. Rules 29, 40, I, and 3 separate ENZ and nonENZ from other types remarkably with few errors. The Rule 29 is a strong discriminator to classify ENZ from other types completely.

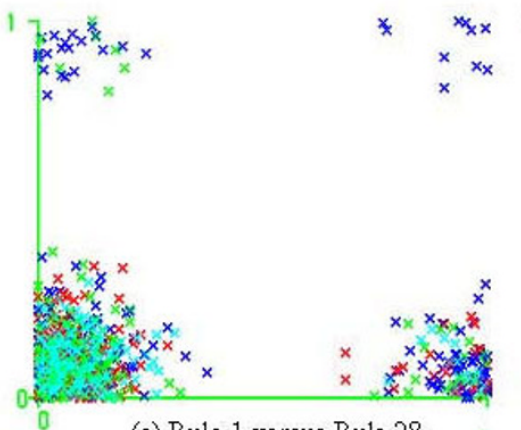

(a) Rule 1 versus Rule 28

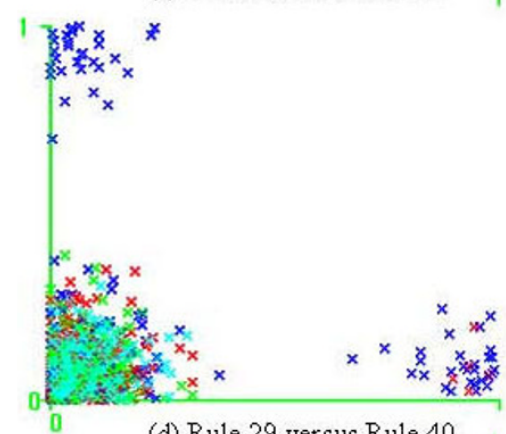

(d) Rule 29 versus Rule 40

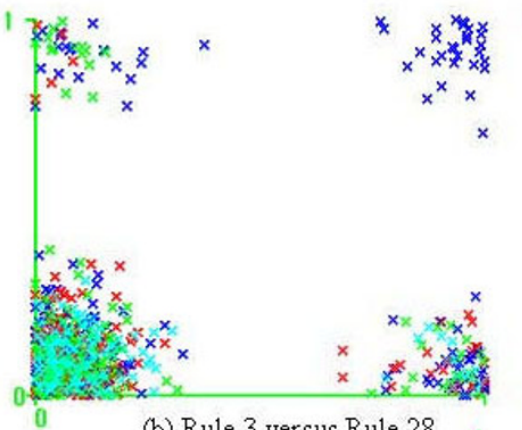

(b) Rule 3 versus Rule 28

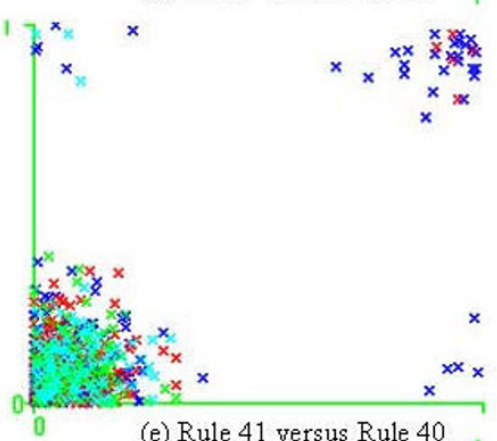

(e) Rule 41 versus Rule 40

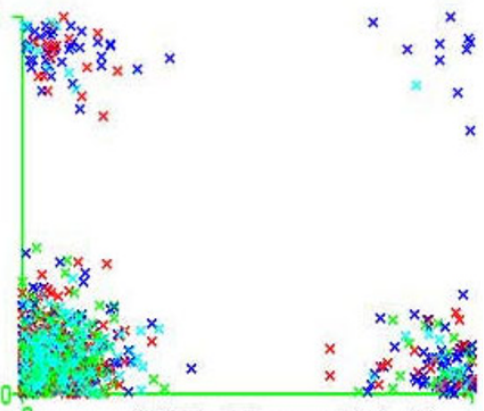

(c) Rule 38 versus Rule 28

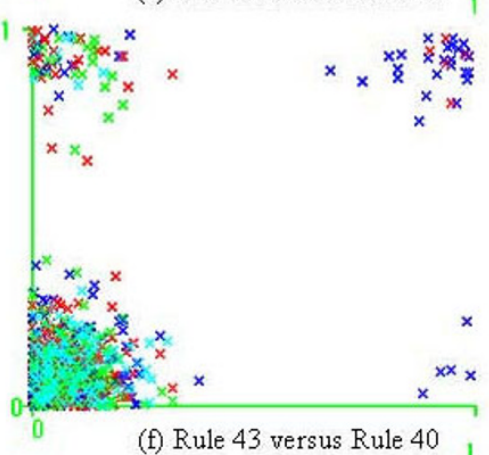

(f) Rule 43 versus Rule 40

\section{Figure 3}

2D plots for pairs of association rules. These plot data points by pairs of association rules. $X$ and $Y$ axes are a pair of rules and each of them have two boolean values. 0 represents negative data points not meeting with a rule of each axis and $I$ represents for positive data points meeting with the rule. The data points on the upper left corner meet a rule used for $Y$ axis and the data points on the down right corner meet a rule used for $X$ axis. The points on the upper right corner meet with both rules used for $X$ and $Y$ axes. Plots in Figure 3(a), (b), and (c) characterize distribution of inhibitors in enzyme-inhibitors interactions. Rule 28 is used for $X$ axis in plots (a), (b) and (c). Rules I, 3 and 38 are used for the $Y$ axis in those plots. (a) represents an example for a pair of rules both including SSE information (e.g. helix and loop content). (b) and (c) show examples for combination of SSE content information (Rule 28: "Nohelix ") with other properties (e.g. SCOPClass, number of atoms and etc.). Plot (b) (Rule 3 versus Rule 28) is identical to the plot generated by Rule 29. Enzymes interacting with a group of inhibitors characterized by (a), (b), and (c) are featured by in Figure 3(e), and (f). Enzymes and inhibitors described by Rules 40 and 29 respectively are plotted in (d) where there is no point matching with both rules. Plot (d) reflects proper interpretation of association rules regarding interactions between enzymes and inhibitors. 


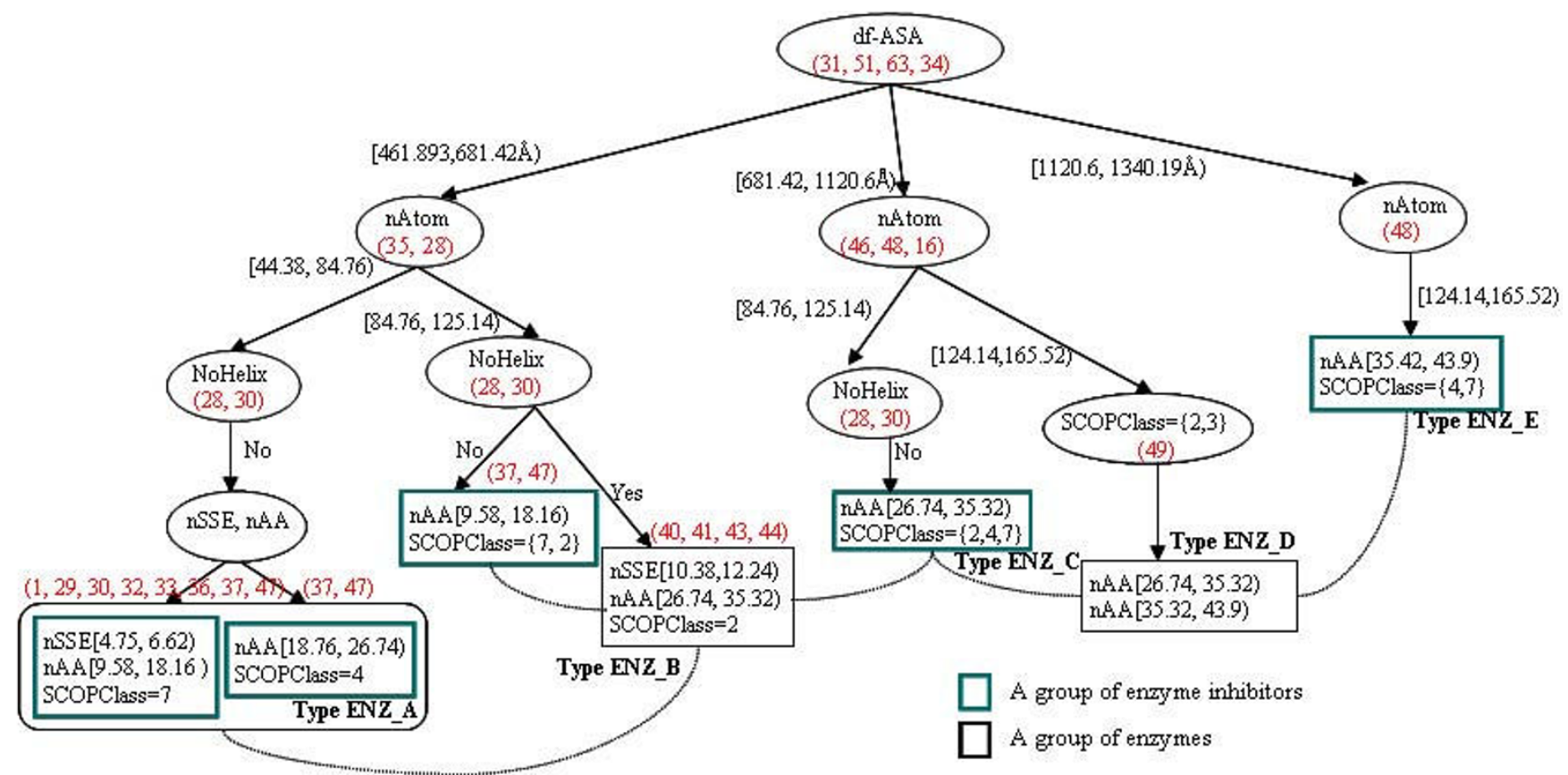

\section{Figure 4}

A hierarchical tree for supporting inference of subtypes. A hierarchical tree drawn from association rules (Table 7) represents different structural groups in ENZ. Enzyme-inhibitor interactions are characterized with size scales of interaction sites (number of atoms and df-ASA) and SSE content information (helix content). These differences of structural groups result in subtypes of PPIs. Letters in red are identifiers of rules (Tables 6 and 7) to split branches of a tree. Dashed lines show interaction between enzymes and inhibitors in different subtypes.

Table 8: PART rules generated by decision trees using $C 4.5^{a}$

\begin{tabular}{|c|c|c|c|c|c|}
\hline$\#^{b}$ & Rules discovered by C 4.5 Decision Tree & Type & Con & $\begin{array}{c}\text { Sup } \\
\text { P }\end{array}$ & Corresponding rules ${ }^{c}$ \\
\hline 5 & $\begin{array}{l}\text { AVGASA }>68.73025 \text { AND nAtom }>60 \text { AND LCS }>2.61 \text { AND Strand } \leq 32.857 \text { AND } \\
\text { SCOPClass }=7\end{array}$ & ENZ & I & 0.03 & $35,5,3,36$ \\
\hline $\begin{array}{l}3 \\
8\end{array}$ & $\begin{array}{l}\text { sRatio } \leq 29.411765 \text { AND HH }>0.277096 \text { AND SCOPClass }=2 \text { AND Strand }>16.949 \text { AND } \\
\text { Strand }>21.324 \text { AND nSSE }>10\end{array}$ & ENZ & I & 0.02 & 40,39 \\
\hline 4 & Loop $>50.299$ AND nAtom $>60$ AND Helix $\leq 33.636$ AND AVGASA $\leq 41.137133$ & ENZ & 0.99 & 0.07 & 35,6 \\
\hline $\begin{array}{l}2 \\
7\end{array}$ & $\begin{array}{l}\text { inPro } \leq 2.016077 \text { AND Helix }>48.485 \text { AND LCS }>1.727 \text { AND Strand } \leq 8.57 \text { I AND } \\
\text { SCOPClass }=\text { I AND AVGASA } \leq 53.133\end{array}$ & $\begin{array}{l}\text { nonEN } \\
\quad Z\end{array}$ & I & 0.02 & 8,10 \\
\hline $\begin{array}{l}4 \\
0\end{array}$ & SCOPClass $=$ I AND Strand $\leq 2.26$ & $\begin{array}{l}\text { nonEN } \\
Z\end{array}$ & I & 0.01 & 15 \\
\hline 1 & $\begin{array}{l}\text { nAtom }>189 \text { AND Loop } \leq 66.316 \text { AND nSSE }>\text { I3 AND Helix } \leq 19.481 \text { AND sRatio } \leq \\
80.833 \text { AND inPro }>-1.570 \text { AND LCS }>3.714 \text { AND Loop } \leq 46.7\end{array}$ & HET & I & 0.05 & 20,21 \\
\hline 3 & $\begin{array}{l}\text { nAtom }>212 \text { AND Strand } \leq 10.738 \text { AND nSSE }>13 \text { AND inPro }>-1.476973 \text { AND nAtom }> \\
384\end{array}$ & HET & $\mathrm{I}$ & 0.05 & $20,18,19$ \\
\hline $\begin{array}{l}3 \\
4\end{array}$ & SCOPClass $=3$ AND Helix $>18.421$ & HOM & $\mathrm{I}$ & 0.02 & 25 \\
\hline $\begin{array}{l}1 \\
5\end{array}$ & $\mathrm{HH}>0.433$ AND AVGASA $>55.984$ AND nAA $\leq 34$ & HOM & I & 0.01 & 27 \\
\hline
\end{tabular}

a: A total of 44 rules produced by a decision tree using C4.5 algorithm in WEKA machine learning library; b\#: PART rule identifier;

cCorresponding rules: Association rule identifiers (Tables 6, 7 and 8) corresponding to a PART rule. 
Table 9: Representative examples of overlapping association rules

\begin{tabular}{|c|c|c|c|c|c|c|c|}
\hline$\#^{a}$ & $\#^{b}$ & Rule descriptionc & Types $^{d}$ & Confe & Supp $f$ & Confg & Supp ${ }^{h}$ \\
\hline 52 & 43 & If $84.76 \leq$ nAtom $<125.14$ AND SCOPClass $=2$ & ENZ' OR nonENZ² & 0.408 & 0.042 & 0.306 & 0.032 \\
\hline 53 & 35 & If $44.38 \leq$ nAtom $<84.76$ AND $461.83 \leq$ df-ASA $<681.42$ & ENZI OR nonENZ² & 0.396 & 0.058 & 0.252 & 0.037 \\
\hline 54 & 48 & If $35.32 \leq \mathrm{nAA}<43.9$ AND $125.14 \leq \mathrm{nAtom}<165.52$ & ENZ' OR nonENZ² & 0.323 & 0.032 & 0.376 & 0.037 \\
\hline 55 & 46 & If $84.76 \leq$ nAtom $<125.14$ AND $681.42 \leq$ df-ASA $<901.01$ & ENZ' OR nonENZ² & 0.252 & 0.032 & 0.336 & 0.042 \\
\hline 56 & 26 & If $3.17 \leq$ LCS $<3.6$ & HET' OR HOM² & 0.357 & 0.037 & 0.337 & 0.035 \\
\hline
\end{tabular}

Examples of overlapping rule are selected from Tables 6 and 7.

a\# Rule identifier;

b\#: Rule identifier in Tables 6 and 7;

Rule descriptionc: The body of overlapping rules between the two types;

'Types: PPI Type' and Type ${ }^{2}$ having overlapping rules in common;

e, gCon f: Confidences of overlapping rules for Type ${ }^{1}$ and $T y p e^{2}$ respectively;

f, hSupp: Supports of overlapping rules for Typel and Type ${ }^{2}$ respectively.

$65)$ to derive subtypes. Hence, the comparative analysis of association rules was presented for ENZ.

\section{Comparison of Association Rules to PART Rules}

To improve our understanding of the association rules discovered, we compared PART rules produced from a decision tree built using $\mathrm{C} 4.5$ over our properties with the association rules. There were a total of 44 PART rules generated and their average confidence and support were 0.99 and 0.02 respectively. We have collected a representative list of PART rules in Table 8. In the comparison of the association rules with PART rules, PART rules are more complicated with the composition of more predicates in rule bodies than those in association rules. Typically, one PART rule corresponds to more than $2 \sim 3$ association rules (Table 8 ). Both rules provided quantitative descriptions. However, property values in PART rules represent split points for classification and are not represented by intervals of quantitative values. Some PART rules (Rules 1, 3 and 38 in Table 8) including identical properties with different split points in the same rule bodies were not clear enough to determine decision boundaries of properties. These limit the readability and understandability of PART rules whilst the association rules were simple enough to be interpreted by users. It was also possible with association rules to support the comparative analysis of rules between different PPI types as we inferred the possibility of subtypes and relative information by comparison of size scales of interaction sites in ENZ. A set of association rules discovered by ARM comprises mostly weak rules together with a small number of strong rules. On the contrary, most PART rules consist of a number of very strong rules which have the highest confidences and low supports.

One of the most notable differences between association rules and PART rules is in how to handle overlapping rules between different types. If two different interaction types are predicted from the identical head of a rule, these are called overlapping rules. There were 99 such cases out of a total of 157 rules (Table 3). Their distribution is illus- trated in Supplementary Figure Nine [see Additional file 2]. Table 9 shows representative examples of overlapping rules. Examination of the overlapping rules shared by ENZ and nonENZ indicated that these types are similar in terms of df-ASA, nAtom, and nAA (Table 9) differentiated by combination with the rest of properties such as SSE content, average length of consecutive residues, size ratio, and hydrophobicity. PART rules are unique cross PPI types.

\section{Conclusion}

We have developed a classification method that categorizes each PPI into one of four different types using association rule based classification (ARBC). The application of association rule mining over 354 known PPI domains using 14 properties yielded a total of 157 rules, which in turn discriminated the features of interaction sites for different PPI types and were used to generate a classification model to predict PPI types. Our ARBC approach performed competitively compared with conventional methods applied directly to the property values: for example, the work in [12] reported an accuracy of $91.8 \%$ for the classification of three types of interactions by directly applying SVM. Although it is not possible to make a direct comparison of their method with ours due to heterogeneity of the data set, this suggests that the processes of association rule generation and subsequent pruning do not incur a loss of relevant information. At the same time, our results demonstrated that we were able to considerably improve the accuracy of the prediction of PPI types through the use of structural domain information for the description of interaction interfaces, and also the use of secondary structure content. Although SSE content alone could not classify interaction sites with high accuracy, its incorporation with other properties improved the accuracy of classification.

Our approach based on ARBC has a clear advantage over conventional methods because results are reported in terms of rules that are a quantitative description of properties and 
hence their interpretation is straightforward and simple. Thus, biologists can easily judge if a discovered rule is interesting or not. Analysis of common and unique properties together is a unique feature of our approach, unlike conventional classification methods which typically capture unique properties only. Common rules capture those properties which are common between PPI types. In particular enzyme inhibitor (ENZ) and non-enzyme inhibitor (nonENZ) interactions, both being non-obligate or transient, share more properties in common than with other types. As we have demonstrated, all these features produce descriptive rules, enabling their simple and powerful interpretation. We observed that the property distributions of homo-obligate interactions are similar to those of heteroobligate interactions but distinct from those of non-obligate interactions. We found that obligate interactions have larger and more hydrophobic interaction sites than nonobligate interactions. Hydrophobic residues including Leu, $\mathrm{Ala}$, and Val were found more frequently in obligate interactions whilst polar residues including Ser and Gly were present in non-obligate interactions. Charged residues (Glu, Asp, Lys, and Arg) were seen frequently in all interaction types. On the basis of a detailed analysis of association rules, it was observed that interactions between enzymes and their inhibitors were separated into several different structural subgroups. This may lead to the possibility of different subtypes of PPIs being involved in transient interactions. Our findings based on the interpretation of association rules are consistent with the description of obligate complexes in previous studies $[17,12]$.

In future work we plan to improve our approach by incorporating additional properties such as energy functions and electric potentials for the generation of more accurate and meaningful association rules. The unique contribution of our work is the development of a novel methodology that analyzes specificities and commonalities for interaction types, and we intend to extend this to the prediction of interaction partner and interaction sites.

\section{Authors' contributions}

SHP developed the concept and the method under the supervision of DRG. JAR carried out classification and participated in drafting the paper. SK interpreted the results for the point of view of a biologist. JWK developed the web application. SHP drafted the paper, JAR, DRG and SK finalized the draft. All authors read and approved the final manuscript.

\section{Additional material}

\section{Additional file 1}

Calculation of residue propensity. A table shows AA propensity for 20 amino acids and a equation represents the calculation of residue propensity.

Click here for file

[http://www.biomedcentral.com/content/supplementary/14712105-10-36-S1.pdf]

\section{Additional file 2}

Association rules. A set of association rules discovered for all types presents and rules are sorted by Type and I.

Click here for file

[http://www.biomedcentral.com/content/supplementary/14712105-10-36-S2.pdf]

\section{Additional file 3}

Distribution of dom-face properties. The figures represent the statistical distributions of dom-face properties for four PPI types.

Click here for file

[http://www.biomedcentral.com/content/supplementary/1471-

2105-10-36-S3.pdf]

\section{Acknowledgements}

We are grateful to Dr K. R. Lee at SSU for helpful discussions. We thank Dr. J. R. Bradford and Prof. D. R. Westhead at University of Leeds, H. Neuvirth and G. Schreiber at Weizmann Institute of Science for providing their data sets. SHP was supported by the Korea Research Foundation Grant funded by the Korean Government(KRF-2005-2 I4-E00050). JAR has been supported by the Programme $\mathrm{Al} \beta \mathrm{an}$, the European Union Programme of High level Scholarships for Latin America, scholarship E04D034854CL. SK was supported by Soongsil University Research Fund.

\section{References}

I. Berman HM, Westbrook J, Feng Z, Gilliland G, Bhat TN, Weissig H, Shindyalov IN, Bourne PE: The Protein Data Bank. Nucl Acids Res 2000, 28:235-242.

2. Vakser IA: Protein-Protein Interfaces Are Special. Structure 2004, 1 2:910-912.

3. Zhou HX, Qin S: Interaction-site prediction for protein complexes: a critical assessment. Bioinformatics 2007, 23(I7):2203-2209.

4. Jones S, Thornton JM: Analysis of protein-protein interaction sites using surface patches. J Mol Biol 1997, 272:121-132.

5. Valdar WS, William JM: Protein-protein interfaces: Analysis of amino acid conservation in homodimers. Proteins 200I, 42(I): $108-124$.

6. Neuvirth H, Raz R, Schreiber G: ProMate: a structure based prediction program to identify the location of protein-protein binding sites. J Mol Biol 2004, 338:181-199.

7. Davis FP, Sali A: PIBASE: a comprehensive database of structurally defined protein interfaces. Bioinformatics 2005, 2I(9): 190I-1907.

8. Livingstone CD, Barton GJ: Protein sequence alignments: a strategy for the hierarchical analysis of residue conservation. Computer Applications in the Biosciences 1993, 9(6):745-756.

9. Bock JR, Gough DA: Predicting protein-protein interactions from primary structure. Bioinformatics 200I, I 7(5):455-460.

10. Koike A, Takagi T: Prediction of protein-protein interaction sites using support vector machines. Protein Eng Des Sel 2004, I7(2): 165-173.

II. Bradford JR, Westhead DR: Improved prediction of protein-protein binding sites using a support vector machines approach. Bioinformatics 2005, 2 I (8): I 487- 1494.

12. Zhu H, Domingues FS, Sommer I, Lengauer T: NOXclass: prediction of protein-protein interaction types. BMC Bioinformatics 2006, 7:27.

13. Chen XW, Liu M: Prediction of protein-protein interactions using random decision forest framework. Bioinformatics 2005 , 2 I(24):4394-4400.

14. Jansen R, Yu H, Greenbaum D, Kluger Y, Krogan NJ, Chung S, Emili A, Snyder M, Greenblatt JF, Gerstein M: A Bayesian Networks Approach for Predicting Protein-Protein Interactions from Genomic Data. Science 2003, 302(5644):449-453.

15. Chothia C, Janin J: Principles of protein-protein recognition. Nature 1975, 256:705-708.

16. Jones S, Thornton JM: Principles of protein-protein interactions. Proc Natl Acad Sci USA 1996, 93:13-20. 
17. Nooren I, Thornton J: Diversity of protein-protein interactions. EMBO J 2003, 22(14):3486-3492.

18. Gunasekaran K, Tsai CJ, Nussinov R: Analysis of ordered and disordered protein complexes reveals structural features discriminating between stable and unstable monomers. J Mol Biol 2004, 34 I(5): | 327-4I.

19. De S, Krishnadev O, Srinivasan N, Rekha N: Interaction preferences across protein-protein interfaces of obligatory and non-obligatory components are different. BMC Struct Biol 2005, 5:.

20. Mintseris J, Weng Z: Structure, function, and evolution of transient and obligate protein-protein interactions. Proc Natl Acad Sci USA 2005.

21. Liu B, Hsu W, Ma Y: Integrating Classification and Association Rule Mining. KDD 1998:80-86 [http://www.aaai.org/Library/KDD/ 1998/kdd98-012.php].

22. Li W, Han J, Pei J: CMAR: Accurate and Efficient Classification Based on Multiple Class-Association Rules. ICDM 2001:369-376 [http://www2.computer.org/portal/web/csdl/doi/ 10.1 109/ICDM.2001.98954I]. IEEE Computer Society

23. Andreeva A, Howorth D, Brenner SE, Hubbard TJ, Chothia C, Murzin $A G$ : SCOP database in 2004: refinements integrate structure and sequence family data. Nucleic Acids Res 2004, 32(32(Database issue)):D226-D229.

24. Wojcik J, Schachter V: Protein-protein interaction map inference using interacting domain profile pairs. Bioinformatics 200I, 17:S296-305.

25. Guharoy $M$, Chakrabarti P: Secondary structure based analysis and classification of biological interfaces: identification of binding motifs in protein protein interactions. Bioinformatics 2007, 23(I 5): 1909-1918.

26. Sanner MF, Olson AJ, Spehner JC: Reduced surface: An efficient way to compute molecular surfaces. Biopolymers 1996, 38(3):305-320.

27. Fauchere J-L, Pliska VE: Hydrophobic parameters $\mathbf{p}$ of amino acid side chains from partitioning of $\mathrm{N}$-acetyl-amino-acid amides. Eur J Med Chem 1983, 18:369-375.

28. Kabsch W, Sander C: Dictionary of protein secondary structure: pattern recognition of hydrogen-bonded and geometrical features. Biopolymers 1983, 22(12):2577-637.

29. Agrawal R, Srikant R: Fast Algorithms for Mining Association Rules in Large Databases. VLDB 1994:487-499 [http://por tal.acm.org/citation.cfm?id=672836].

30. Viksna J, Gilbert D, Torrance GM: Protein structure comparison based o $n$ profiles of topological motifs: a feasible way to deal with information from negative examples. German Conference on Bioinformatics 2003:159-165.

31. Witten IH, Frank E: Data Mining: Practical machine learning tools and techniques 2nd edition. 2005 [http://www.cs.waikato.ac.nz/ ml/weka/ book.html]. San Francisco: Morgan Kaufmann

32. Hall MA: Correlation-based Feature Selection for Machine Learning. Tech rep 1998.

33. Quinlan R: C4.5: Programs for Machine Learning 1993 [http:// www.springerlink.com/content/v986m I562062hk5I/]. San Mateo, CA: Morgan Kaufmann Publishers
Publish with Bio Med Central and every scientist can read your work free of charge

"BioMed Central will be the most significant development for disseminating the results of biomedical research in our lifetime. "

Sir Paul Nurse, Cancer Research UK

Your research papers will be:

- available free of charge to the entire biomedical community

- peer reviewed and published immediately upon acceptance

- cited in PubMed and archived on PubMed Central

- yours - you keep the copyright
BioMedcentral 